\title{
A Joint Routing and Time-Slot Assignment Algorithm for Multi-Hop Cognitive Radio Networks with Primary-User Protection
}

\author{
H. Chen, Q. Du, P. Ren
}

\author{
Hao Chen \\ 1. Department of Information and Communication Engineering, \\ Xi' an Jiaotong University, Xi' an, Shaanxi,710049, China \\ 2. State Key Laboratory of Integrated Services Networks, \\ Xidian University, Xi'an, Shaanxi, 710071, China \\ E-mail: js.sq.chenhao@stu.xjtu.edu.cn

\section{Qinghe Du, Pinyi Ren} \\ Department of Information and Communication Engineering, \\ Xi'an Jiaotong University, Xi'an, Shaanxi,710049, China \\ E-mail: \{duqinghe, pyren\}@ mail.xjtu.edu.cn
}

\begin{abstract}
:
Cognitive radio has recently emerged as a promising technology to improve the utilization efficiency of the radio spectrum. In cognitive radio networks, secondary users (SUs) must avoid causing any harmful interference to primary users (PUs) and transparently utilize the licensed spectrum bands. In this paper, we study the PUprotection issue in multi-hop cognitive radio networks. In such networks, secondary users carefully select paths and time slots to reduce the interference to PUs. We formulate the routing and time-slot assignment problem into a mixed integer linear programming (MILP). To solve the MILP which is NP-Hard in general, we propose an algorithm named RSAA (Routing and Slot Assignment Algorithm). By relaxing the integral constraints of the MILP, RSAA first solves the max flow from the source to the destination. Based on the max flow, RSAA constructs a new network topology. On the new topology, RSAA uses branch and bound method to get the near optimal assignment of time slots and paths. The theoretical analyses show that the complexity of our proposed algorithm is $O\left(N^{4}\right)$. Also, simulation results demonstrate that our proposed algorithm can obtain near-optimal throughputs for SUs.

Keywords: Cognitive Radio Networks; Primary-user Protection; Joint Routing and Time-slot Assignment.
\end{abstract}

\section{Introduction}

The rapid growth in the number of wireless applications such as WiFi, WiMAX, 3G et al. leads to a big radio spectrum shortage. Recent studies by the Federal Communications Commission (FCC) highlight that the average utilizations of some licensed spectrum bands allocated through the current static frequency spectrum assignment policies vary between $15 \%$ and $85 \%$ [1]. To make sufficient use of the spectrum resources in the environment, the notion of cognitive radio (CR) was proposed by Dr. Joseph Mitola in 1999 [2]. In cognitive radio networks, nodes are allowed to sense and explore a wide range of the frequency spectrum and identify currently underutilized spectrum blocks for data transmission. CR can transparently exploit the licensed spectrum bands and is widely considered as the technique for the next generation of wireless communication [3].

To maximize the advantages of cognitive radio networks (CRNs) EŹit is necessary to update the physical layer, media access control (MAC) layer and network layer of the traditional wireless communication system. After the concept of CR was proposed, many studies on spectrum sensing and MAC protocol 
design have been conducted and a lot of progress has been made [4] [5] [6]. The aim of spectrum sensing is to find the spectrum holes in the CRNs and the MAC protocol is to select the one-hop optimal spectrum bands for SUs' transmitting. However, Khalife et al. indicated that MAC protocol which gave optimal solutions in a single hop configuration may become largely inefficient in a multi-hop scenario and it was of great importance to design cross-layer protocols capable of scheduling, spectrum selecting and routing [7]. Cesana et al. in [8] pointed out the challenges of routing in cognitive radio networks: any routing solutions designed for multi-hop CRNs must be highly coupled to the entire cognitive cycle of spectrum management and the routing module should be able to make fast route maintenance at the sudden appearance of PUs. The authors of [9] extended the routing solution in multi-channel multi-radio networks (MCMRNs) to CRNs and proposed a layered graph framework to address channel assignment and routing jointly. Hou et al. in [10] [11] illustrated the difference about routing in MCMRNs and CRNs. In CRNs the radios could send packets over non-contiguous frequency bands and the authors proposed a mixed integer non-linear programming (MINLP) model to minimize the required networkwide radio spectrum resources. Filippini et al. in [12] proposed a minimum maintenance cost routing for cognitive radio networks. The authors formulated the maintenance cost problem to be an integer optimization model and by carefully selecting routing metrics the authors designed a heuristic distributing algorithm.

Among all the above routing solutions the accurate information about spectrum availability sensed by the physical layer is crucial for the routing module. So these routing solutions make severe demand on the spectrum sensing module of CR nodes. To ease this demand, in [13] Chowdhury et al. proposed a routing solution which avoided harmful interference to $\mathrm{PU}$ receivers by designing proper routing metrics. In that solution, each time SUs chose the path that passed through regions having minimum overlap with PUs' transmission coverage areas. In [14] Ding et al. proposed a distributed algorithm to maximize the capacity of links without generating harmful interference to other users by performing joint routing, dynamic spectrum allocation and scheduling. In [15] Xie et al. proposed a geometric approach for relay selections which avoided causing harmful interference to PUs in CRNs. Each time the approach selected the best channels available to transmit data to the nearest neighbors or the farthest ones greedily. In [16] the authors Zhou et al. gave a mathematical model aiming at minimizing the interference to PUs. By relaxing the model's constraint conditions, the authors transformed the original optimization problem to a linear programming model and gave a joint channel assignment and path selection algorithm.

In spectrum sharing multi-hop CRNs, to guarantee the PUs' priority on the licensed spectrum bands SUs must not generate harmful interference to PUs. In this paper, to make sufficient use of spectrums we design the routing module of SUs through joint routing and time-slot assignments. Firstly, we exploit the Protocol Model [17] which describes the conditions of successful transmission between two nodes and abstract the routing and time-slot assignment problem to be a kind of mixed integer linear programming (MILP) model. In the MILP model, our objective is to maximize the throughput of SUs and our constraints are avoiding harmful interference to PUs and eliminating SUs' conflicts caused by concurrent transmissions. Then, to get an approximate solution of the MILP which is a NP-Hard problem we propose a near optimal joint routing and time-slot assignment algorithm named RSAA. The theoretical analysis shows that the complexity of RSAA is $O\left(N^{4}\right)$. The simulation results demonstrate that RSAA can obtain near optimal throughput. What is more, through the simulation results we analyze the effect of node density and slot periods on the throughput of multi-hop CRNs. 


\section{Network Model and Problem Formulation}

\subsection{Network Model}

In 2004, the FCC proposed to allow unlicensed wireless devices to utilize television channel frequencies under the precondition of causing no harmful interference to PUs. Under this proposal, SUs could use the underutilized broadcasting TV spectrum bands for multi-hop communications. In multihop CRNs, TDMA is very necessary for the avoidance of conflicts among SUs and the reduction of interference to PUs. In the current WLANs, nodes use CSMA/CA to avoid access conflicts. However, CSMA/CA cannot assure that SUs cause no harmful interference to PUs. Hence it may be not suitable for spectrum sharing CRNs.

In this paper, we consider a cognitive Ad Hoc network consisting of $P$ PUs, $N$ SUs and a Cognitive Scheduling Center (CSC). The CSC is able to access the data base of PUs [8] and gathers the information about the PUs' locations and interference thresholds. The interference threshold is defined as the highest interference power which a PU can tolerate. Also the CSC is designed to be able to collect the information about SUs' locations and the transmitting power via existing communication networks like GSM. After gathering all these information, the CSC computes the optimal routing and time-slot assignments and schedule SUs' access to licensed spectrum by delivering these messages to the corresponding nodes. The network architecture is shown in Fig. 1.

As is shown in Fig. 1, the TV receivers act as PUs and have priority to use the spectrum $f$.The CSC coordinates and schedules SUs to utilize the band $f$ transparently. For example, the CSC assigns the path and slot solution $s \stackrel{1}{\rightarrow} a \stackrel{2}{\rightarrow} b \stackrel{3}{\rightarrow} c \stackrel{1}{\rightarrow} e \stackrel{2}{\rightarrow} d$ for source node $s$ and destination node $d$. The symbol " $a \stackrel{1}{\rightarrow} b$ " represents that node $a$ transmits to node $b$ in time slot 1 . Although the path $s \rightarrow a \rightarrow g \rightarrow e \rightarrow d$ is of less hops and much more throughput, the CSC does not choose it because node $g$ causes harmful interference to PUs.This paper focuses on finding the optimal routing and time-slot assignment which maximizes the SUs' throughput while avoiding harmful interference to PUs.

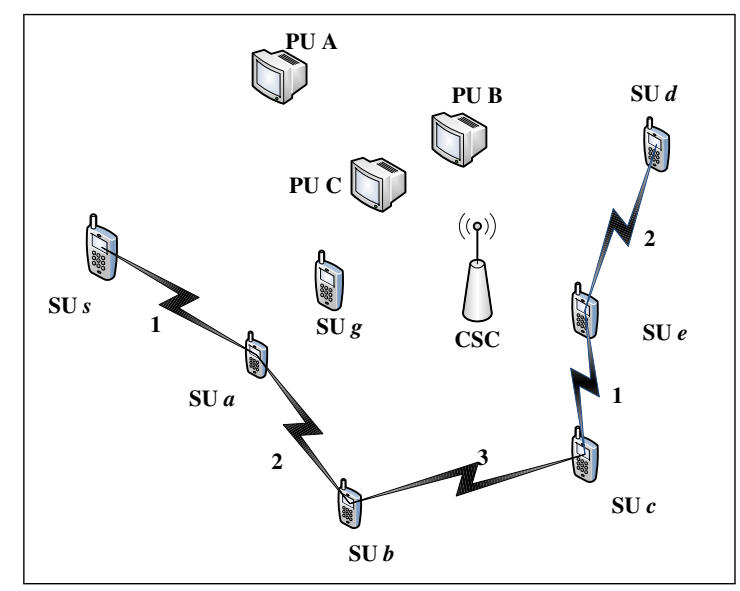

Figure 1: system model

\subsection{Problem Formulation}

To describe the network mathematically, we first model the successful transmitting conditions among nodes and introduce binary integral variables $x_{i j t}$, where $x_{i j t}=1$ indicates that SU node $i$ sends packets to SU node $j$ in slot $t$; otherwise $x_{i j t}=0$. That is,

$$
x_{i j t}=\left\{\begin{array}{l}
1, S U i \text { send packets to } S U j \text { in slot } t \\
0, \text { otherwise }
\end{array}\right.
$$


We assume that all the SUs' radios use the same transmitting power $Q$ and the successful transmitting range among SUs are $R_{T}$. Let $A_{i}$ denote the neighbor set of node $i$. So we have

$$
A_{i}=\left\{j \mid d_{i j}<R_{T}\right\}
$$

where $d_{i j}$ denotes the Euclid distance between node $i$ and node $j$.

Let $I_{i}$ denote the interfered node set of node $i$, and $R_{I}$ denote the interference range among SUs. So we have

$$
I_{i}=\left\{j \mid d_{i j}<R_{I}\right\}
$$

Note that node $i$ cannot transmit to multiple nodes at the same time. We have

$$
\sum_{q \in A_{i}} x_{i q t} \leqslant 1
$$

Due to potential interference among nodes in the network, if node $i$ uses slot $t$ for transmitting data to node $j \in A_{i}$, then any other nodes which conflict with node $j$ should not use this slot. So we have

$$
x_{i j t}+x_{p q t} \leqslant 1, p \in I_{j}, p \neq i, q \in A_{p}
$$

This is the difference between our model with the model in [9][10], where the authors state the conflict constraint as

$$
x_{i j t}+\sum_{p \in I_{j}, p \neq i, q \neq j} x_{p q t} \leqslant 1
$$

It is important to understand that in the conflict constraint (5), two links which interfere with $x_{i j t}$ but do not interfere with each other can access the channel at the same time slot. Constraint (4) is the node's successful sending condition and constraint (5) is the node's successful receiving condition. Under the constraint (4) and (5), nodes in the network can transmit data without conflicting with each other.

To guarantee that two nodes transmit data without bit error, the flow rates on each link must not exceed the link's capacity. Let $f_{i j t}$ denote the flow rate between node $i$ and node $j$ at time slot $t$ and $g_{i j}$ denote the channel gain between node $i$ and node $j$. We have

$$
f_{i j t} \leqslant x_{i j t} B \log _{2}\left(1+\frac{g_{i j} Q}{\eta}\right)
$$

where $B$ denotes the bandwidth of spectrum band $f$ and $\eta$ denotes the noise power in the environment. Note that the denominator inside the $\log$ function contains only $\eta$. This is because the interference constraint (5) assures that the interference power received by the SU's receiver is negligible and this helps to simplify our model to be a linear model.

What's more, to make sure that no drop of packet happens at the intermediate nodes, the aggregate data rates in the slot period should meet the flow conservation constraint. i.e.

$$
\sum_{t=1}^{T} \sum_{j \in A_{i}} f_{i j t}=\sum_{t=1}^{T} \sum_{i \in A_{p}} f_{p i t}
$$

where $T$ denotes the period of scheduling slots of the network.

To make sure that all the transmitting power of SUs detected at PUs does not exceed PUs' threshold $I n_{T}$, we have the following constraints

$$
\sum_{i=1}^{N} x_{i j t} g_{i k} Q \leqslant \operatorname{In}_{T}, j \in A_{i}, k \in P,
$$


where $P$ denotes the set of PUs. In practice the value of $I_{T}$ depends on the sensitivity of PU receivers as well as the noise power in the environment.

Note the average value of SUs' throughput $S$ is the ratio of aggregate amount of data to the time slots, so we have

$$
S=\frac{1}{T} \sum_{t=1}^{T} \sum_{i \in A_{s}} f_{s i t}
$$

where $s$ denotes the source node.

When there are a number of source-destination pairs in the network, we can introduce one more virtual source-destination pair and simplify the network to be a single source-destination pair network. In such networks, our aim is to find the optimal routing and time-slot assignments which maximize the throughput of SUs. Mathematically, we have the following optimization problem.

$$
\begin{gathered}
\max \left\{\frac{1}{T} \sum_{t=1}^{T} \sum_{i \in A_{s}} f_{s i t}\right\} \\
\text { s.t. }\left\{\begin{array}{l}
\sum_{q \in A_{i}} x_{i q t} \leqslant 1 \\
x_{i j t}+x_{p q t} \leqslant 1, p \in I_{j}, p \neq i, q \in A_{p} \\
f_{i j t} \leqslant x_{i j t} B \log _{2}\left(1+\frac{g_{i j} Q}{\eta}\right) \\
\sum_{t=1}^{T} \sum_{j \in A_{i}} f_{i j t}=\sum_{t=1}^{T} \sum_{i \in A_{p}} f_{p i t} \\
\sum_{i=1}^{N} x_{i j t} g_{i k} Q \leqslant I n_{T}, j \in A_{i}, k \in P, 0 \leqslant t \leqslant T \\
x_{i j t}=0 \text { or } 1 \\
f_{i j t} \geqslant 0
\end{array}\right.
\end{gathered}
$$

Note that the above optimization problem has both integral and continuous variables and the constraint conditions are all linear. So it is a kind of MILP problem. In the above MILP problem, the optimization variables consists of continuous variables $f_{i j t}$ and binary variables $x_{i j t}$, while $B, T, Q, \eta$ and $\operatorname{In}_{T}$ are all constant.

\section{Routing and Time-Slot Assignment Algorithm}

The above optimization problem is a kind of MILP problem, which is NP-Hard in general [18]. To solve the MILP problem, Yuan et al. in [16] proposed a greedy algorithm which relaxed the integral constraints and then simplified the MILP to be a linear programming (LP) problem. After solving the LP problem, the algorithm fixed the binary variables in the descending order of their relaxed values one by one. Although the complexity of this algorithm is equal to solving just one LP problem, the algorithm cannot guarantee that the solution is a feasible flow and that the result meets the interference constraints (4) (5) absolutely. Hou et al. in [10] proposed the Sequential Fixing (SF) algorithm which solved $O(N)$ LP problems iteratively to fix the binary variables. Another method to solve MILP is to replace the integral constraint to be the following constraint

$$
x_{i j t}\left(x_{i j t}-1\right)=0 .
$$

Based on constraint (13) we can transform MILP to be quadratic programming (QP). But we still cannot get optimal solution under polynomial complexity because constraint (13) is non-convex.

Sherali et al. in [18] pointed out that to solve the MILP problem one should exploit the problem's inherent special structures in the process of model formulations and in algorithmic developments. Taking 
a closer look at our MILP problem we can find that if we relax the integral constraint (1) to be continuous constraint

$$
0 \leqslant x_{i j t} \leqslant 1
$$

and neglect the interference constraint (4)(5). Then the MILP problem will reduce to a kind of max flow problem. So the optimal solutions of MILP are likely to be the subsets of the max flow. Based on this idea, we develop our algorithm RSAA which can solve the MILP problem efficiently.

Let $E$ denote the number of edges and $N$ denote the number of SU nodes in the CRNs. Since the original MILP consists of ET binary variables, to get the optimal solution of the MILP problem we should enumerate $2^{E T}$ combinations of $x_{i j t}$. Once the binary variables are fixed, the original MILP problem is reduced to be the following LP problem.

$$
\begin{gathered}
\max \left\{\frac{1}{T} \sum_{t=1}^{T} \sum_{i \in A_{s}} f_{s i t}\right\} \\
\text { s.t. }\left\{\begin{array}{l}
f_{i j t} \leqslant x_{i j t} B \log _{2}\left(1+\frac{g_{i j} Q}{\eta}\right) \\
\sum_{t=1}^{T} \sum_{j \in A_{i}} f_{i j t}=\sum_{t=1}^{T} \sum_{i \in A_{p}} f_{p i t} \\
\sum_{i=1}^{N} x_{i j t} g_{i k} Q \leqslant I n_{T}, j \in A_{i}, k \in P, 0 \leqslant t \leqslant T \\
f_{i j t} \geqslant 0
\end{array}\right.
\end{gathered}
$$

We denote the new LP problem as LP1 and it consists of ET continuous variables.

In RSAA algorithm, we first neglect the constraint conditions (4)(5)(7) and solve the max flow problem from the source to the destination. The max flow problem can be formulated to be the following LP problem

$$
\begin{gathered}
\max \left\{\frac{1}{T} \sum_{t=1}^{T} \sum_{i \in A_{s}} f_{s i t}\right\} \\
\text { s.t. }\left\{\begin{array}{l}
f_{i j t} \leqslant B \log _{2}\left(1+\frac{g_{i j} Q}{\eta}\right) \\
\sum_{t=1}^{T} \sum_{j \in A_{i}} f_{i j t}=\sum_{t=1}^{T} \sum_{i \in A_{p}} f_{p i t} \\
f_{i j t} \geqslant 0
\end{array}\right.
\end{gathered}
$$

We denote the above LP problem as LP2 and use Push-Pull Flow Algorithm [20] to obtain the max flow $\Phi=\left\{f_{i j t}\right\}$. After getting $\Phi$, we construct a new set of binary variables by

$$
X_{T}=\left\{x_{i j t} \mid f_{i j t}>0, f_{i j t} \in \Phi\right\}
$$

And $X_{T}$ is the new enumeration set of RSAA. We denote $K$ as the number of variables of $X_{T}$, i.e. $K=\left|X_{T}\right|$. When we enumerate the new variables in $X_{T}$, we use constraints (4)(5) as the branch and bound conditions. The complete RSAA algorithm is given in Table 1.

In the procedure of RSAA algorithm, we first construct new searching variables or edges by the solution of the max flow problem and then cut off all the nodes and edges which have nothing to do with the max flow in the network. To solve the MILP in newly constructed network, we take the condition (4) (5) as bound conditions and simplify the MILP problem to be a number of LP1 problems. Also when we solve LP1, we take it as a kind of special max flow problem, and use Dinic algorithm to solve it. As we can see from the algorithm's process, the solution of RSAA always meets all the constraints of the original MILP problem and so we can conclude that the solution of RSAA is a feasible solution to the original MILP problem. 
Table 1: RSAA algorithm

\begin{tabular}{|c|c|}
\hline Steps & Contents \\
\hline Step 1: & $\begin{array}{l}\text { Let the CSC update the PUs' and SUs' locations and compute the SUs' link capacity and } \\
\text { their interference power to PUs. Introduce a virtual source-destination pair and simplify } \\
\text { the network to be a signal source-destination pair network. }\end{array}$ \\
\hline Step 2: & $\begin{array}{l}\text { Set up and solve LP2 and obtain its solution } \Phi \text {. Use the equation (19) and } \Phi \text { to construct } \\
\text { new binary variable set } X_{T} \text {. Sort the new binary variables in the ascending order by slot } \\
\text { index. }\end{array}$ \\
\hline Step 3: & $\begin{array}{l}\text { Initialize the SUs' throughput } S=0 \text {; set the current optimal flow solution as } \Phi^{*}=\emptyset \text {, } \\
\text { temp_i } i=0 \text {. }\end{array}$ \\
\hline Step 4: & $\begin{array}{l}\text { If } t e m p \_i>2^{K} \text {, then the whole algorithm ends, output the optimal throughput } S \text { and } \\
\text { the optimal flow solution set } \Phi^{*} \text {; else transform temp_i into }\left|X_{T}\right| \text { bit binary digits, each } \\
\text { digit represents the assignment of corresponding link and slot. If each digit of } t e m p \_i \\
\text { meets the interference condition (4)(5),then go to Step 5; else temp_i } i e m p \_i+2^{b \_l a s t-1} \text {, } \\
\text { where } b \_l a s t \text { is the smallest digit index in all temp_i's transformed digits which violate } \\
\text { the interference condition (4)(5). Go back to Step } 4 \text {. }\end{array}$ \\
\hline Step 5: & $\begin{array}{l}\text { After getting one combination of }\left\{x_{i j t}\right\} \text {, use Dinic algorithm to solve LP1 and get the max } \\
\text { flow value temp_fval as well as the corresponding flow rates } F_{T}=\left\{f_{i j t}\right\} \text {. In the steps of } \\
\text { Dinic algorithm, when we search the augmenting flows in the layered networks, we select } \\
\text { the augmenting flow according to the ascending order of their interference to PUs. If the } \\
\text { adding of augmenting flow with the minimum interference exceeds } I n_{T} \text {, then augmenting } \\
\text { step of Dinic algorithm ends; else continue to find the other augmenting flows. When } \\
\text { Dinic algorithm ends, if } S<t e m p \_f v a l \text {, then } S=t e m p \_f v a l, \Phi^{*}=F_{T} \text {; else go back to } \\
\text { Step } 4 \text {. }\end{array}$ \\
\hline
\end{tabular}




\section{Performance Analysis of RSAA}

\subsection{Complexity of RSAA}

We now analyze the complexity of RSAA using the random network theory. In the original MILP problem, the complexity of obtaining optimal solution is exponential and we should solve $O\left(2^{|E|}\right)$ linear programming LP1. By reducing the searching variables, the complexity of RSAA algorithm is reduced to be polynomial. In fact, we have the following theorem.

Theorem 1. In the network where the average degree of each node is constant, the complexity of RSAA is $O\left(N^{4}\right)$.

Proof: Let constant $D$ denote the average degree of each node in the network and in practice the value of $D$ is determined by the node density and the node's transmitting range. By the ER model in random network theory [21], the average number of edges in the network is

$$
E=\frac{D N}{2}
$$

Let $L$ denote the average path length from the source node to the destination node, and then the number of nodes in the network has the following expression [21],

$$
N \propto D^{L}
$$

So the average route length can be written as

$$
L=\alpha \frac{\log _{2} N}{\log _{2} D}
$$

where $\alpha$ is a constant. Note that the capacity of each link in random networks is i.i.d. So the maximum throughput we obtain from LP2 are $D C$, where $C$ denotes the average capacity of links. And then we can conclude that the maximum number of paths in the max flow for source to destination is $D$. Hence, the number of newly constructed binary variables is

$$
K=\left|X_{T}\right|=D T L=\alpha T D \frac{\log _{2} N}{\log _{2} D} .
$$

So RSAA need to solve $O\left(2^{K}\right)=O(N)$ linear programming problems LP1. By Dinic algorithm the complexity of solving LP1 is $O\left(N^{3}\right)$. Hence, the total complexity of RSAA algorithm is $O\left(N \bullet N^{3}\right)=$ $O\left(N^{4}\right)$.

From Theorem 1 we can see that both RSAA and SF need to solve $O(N)$ linear programming problems LP1. The difference between them is that RSAA exploits the flow structures of the network to obtain better performance.

\subsection{Optimal Approximation}

Although RSAA restricts the searching space and this restriction may reduce the throughput of SUs, we find that this kind of reduction is almost negligible. In fact we have the following theorem.

Theorem 2. If $\Phi^{*}$ is the optimal solution to the MILP problem and $P_{T}$ is the optimal routing and timeslot assignment, then the intersection set between $P_{T}$ and the RSAA's new constructed searching set $X_{T}$ is not null. 
Proof: We prove this theorem by constructing contradictions. Suppose the intersection set between $X_{T}$ and the new constructed searching set $X_{T}$ is null, i.e. $P_{T} \cap X_{T}=\emptyset$. We denote the optimal flow rate of the MILP as $\Phi^{*}$ and denote the max flow of LP2 as $\Phi$. Because $\Phi^{*}$ is the solution of the MILP, and so $\Phi^{*}$ satisfies all the constraint flow conditions in the MILP. Then $\Phi^{*}$ satisfies all the constraint conditions of LP2. So $\Phi^{*}$ is a feasible augmenting flow to LP2. Because $P_{T} \cap X_{T}=\emptyset$, and this means $P_{T}$ is an independent augmenting path on which we can augment $\Phi^{*}$ to the original max flow. So the optimal solution of LP2 is $\Phi^{*}+\Phi$ and this is contradictory to the fact that $\Phi$ is the optimal solution. So the supposition that the intersection set between $P_{T}$ and the RSAA's new constructed searching set $X_{T}$ is null is false and our theorem is proven.

However, Theorem 2 cannot guarantee that the solution of RSAA is optimal. Only when $P_{T}$ is the subset of $X_{T}$, can we say that the solutions of RSAA are optimal. In fact, we can conclude that $P_{T}$ belongs to the subset of $X_{T}$ with high probability according to Theorem 2. Especially, in the small networks where the max flow consists of only one path, we can conclude that the solution of RSAA will be optimal according to Theorem 2 and the flow conservation condition(8).

\subsection{The Effect of Time-Slots and Conflicts}

In our MILP model, there is a constant $T$ which represents the slot scheduling period of the CRNs. We can see that the existing of $T$ increases the complexity of our algorithm, and this is because $T$ decides the number of variables. In the network layer, the routing module should fix the best slot period according to the network parameters such as the node density and PU's threshold. Note that the minimum slot period should be enough to avoid the conflicts among SUs and the interferences to PUs. So we can get the average minimum value of $T$,

$$
T_{\min }=\max \left\{L Q g^{*} / I n_{T}, L / c\right\}
$$

In the above equation, $c$ is the average number of mutual interference edges and $L$ is the average route length from the source to destination, and $g^{*}$ denotes the average channel gain between two neighbor nodes. In equation (24), the first item means that to connect the source and the destination at least $L Q g^{*} / I n_{T}$ slot periods are needed to guarantee no harmful interference to PUs. The second item means that to avoid the conflicts among SUs at least $L / c$ slot periods are needed. And the equation (14) means that only the slot periods are long enough to avoid harmful interference to PUs and eliminate the conflicts among SUs, the source and destination pair can set up a successful route.

In fact, it is necessary to assure that the slot period is greater than the minimum one, or the slot period will become the bottleneck constraint of SU's throughput. And when the slot periods are smaller than the minimum one the increase of slot period will dramatically increase SUs' throughput. But if the slot periods are greater than the minimum one, the increase of slot period will not necessarily lead to the increase of SUs' throughput. And in this scenario SUs' throughput in CRNs are the tradeoff between the amount of data and the delay as is shown in equation (10). However, if we divide one fixed length of time into a number of slots, we can find that the more slots we divide the fixed time into, the more throughput SUs can obtain. In the best case if we divide the fixed time into infinite slots, and then the solution of MILP will approximate the solution of the relaxed LP where the integral constraint is reduced to be constraint (14).

To describe the effect of conflict on SUs' throughput, we introduce $R$ denoting the ratio of node's interference distance to transmitting distance. i.e.

$$
R=R_{I} / R_{T}
$$

Note that as the ratio $R$ increases the number of conflicting edges in the network will increase. And this means the number of conflict constraint inequality (5) will increase and so the solutions of the MILP will 
decrease. Intuitively, the increase of the ratio $R$ will decrease the concurrent transmissions and cut down SUs' throughput. However, as the ratio $R$ increases, the number of paths from the source to destination will decrease because of the concurrent conflicts. So according to Theorem 2 we can conclude that the solution of RSAA will become closer to the optimal results as the ratio $R$ increases.

From the analysis above, we can find that RSAA algorithm can obtain the near optimal throughput of SUs at the complexity of $O\left(N^{4}\right)$. In the next section we will verify the performance analysis through simulations.

\section{Simulation Results}

In this section, we present simulation results for the proposed RSAA algorithm and compare it to SF algorithm and the enumeration algorithm. Since the enumeration algorithm can obtain optimal solutions, we denote the results of enumeration algorithm as optimal solutions in the following figures. Our simulation scenario is set at the rural and mountainous areas where the TV broadcast spectrum is underutilized and the SUs can transparently use these spectrums without generating harmful interference to PUs. The simulation parameters are shown in Table 2.

Table 2: Simulation Parameters

\begin{tabular}{cc}
\hline Simulation Parameters & Values \\
\hline The topology area & $1000 \times 1000 \mathrm{~m}^{2}$ \\
The distribution of SUs' location & uniform distribution \\
Channel Propagation model & two way ground reflection model \\
The transmitting range of SUs & $250 \mathrm{~m}$ \\
The interfering range of SUs & $300 \mathrm{~m}$ \\
The band width of PUs & $1 \mathrm{MHz}$ \\
The power of noise & $-140 \mathrm{dBW}$ \\
The transmitting power of SUs & $2 \mathrm{~W}$ \\
The number of PU & 1 \\
The location of PU & $(0,0)$ \\
The length of each slot period & $1 \mathrm{~s}$ \\
Simulation times & 200 \\
\hline
\end{tabular}

\subsection{The SUs' throughput vs PU's outage probability}

Fig. 2 shows the outage probability of PUs in the condition that SUs do not take the PU's threshold into consideration. We count a time of outage of PU when PU detects that SUs' interference Power exceeds $I n_{T}$. In Fig. 2 the slot period is set to be 3 and the ratio of interference to transmitting is set to be 6/5. We use software CPLEX to get the optimal solution of MILP.

From Fig. 2 we can find that as the PU's threshold increases the outage probability of PU decreases. Also the outage probability decreases as the node density $\mathrm{Nm}$ in the network decreases. This is because the interference power received by PU is the sum of all SUs' transmitting power and more nodes in the network means more interference. As we can see from Fig. 2, the outage probability is very high and unbearable if SUs do not carefully select paths and slots. So in the networks with high node density, it is very necessary to protect PU from SUs' interference at network layer.

Fig. 3 compares the SUs' throughput in two different scenarios: in one scenario SUs avoid harmful interference to PUs and in the other scenario SUs neglect PU's threshold. From Fig. 3 we can see that SUs' throughput are very sensitive to PU's threshold when SUs take the interference to PU into 


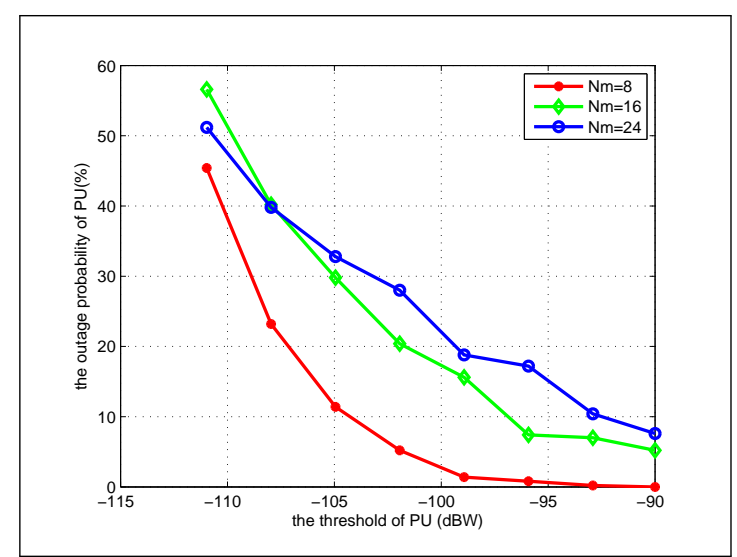

Figure 2: the outage probability of PU as the increase of PU's threshold

consideration. And the throughput obtained from neglecting PU's threshold are the upper bound of those of considering PU's threshold. What's more, Fig. 3 shows that when PU's threshold is low enough, PU's threshold becomes the bottleneck of SUs' throughput. Fig. 2 and 3 demonstrate the performance tradeoff between PUs and SUs in spectrum sharing CRNs. And so our model can offer valuable reference for the design of multi-hop CRNs.

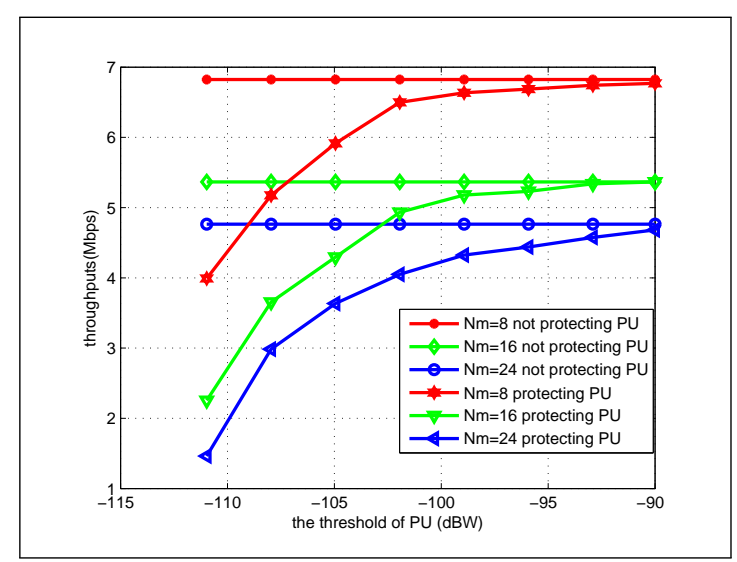

Figure 3: SUs' throughput as the increase of PU's threshold

\subsection{The Effect of Node Density}

Fig. 4 compares SUs' throughput of RSAA algorithm, SF algorithm and the enumerate algorithm. In the simulation, the slot period is 3 and the PU's threshold is $-90 \mathrm{dBW}$. The ratio of interference to transmitting is 6/5. From Fig. 4 we can find that the results of RSAA outperform the results of SF and can obtain $97 \%$ of the optimal throughput for SUs averagely. Especially when the node density is low, RSAA can obtain $99 \%$ of the optimal throughput, while SF just gets 55\% optimal throughput for SUs. This is because when the node density is low, the optimal path will almost surely locate in the searching set $X_{T}$ as is shown in Theorem 2. What's more, Fig. 4 shows that SUs' throughput decrease as the node density increases which is the same with the results in [16]. The reason is that when the node density increases, SUs need more slots which mean more delay to avoid the conflicts among SUs and the interference to PU. 


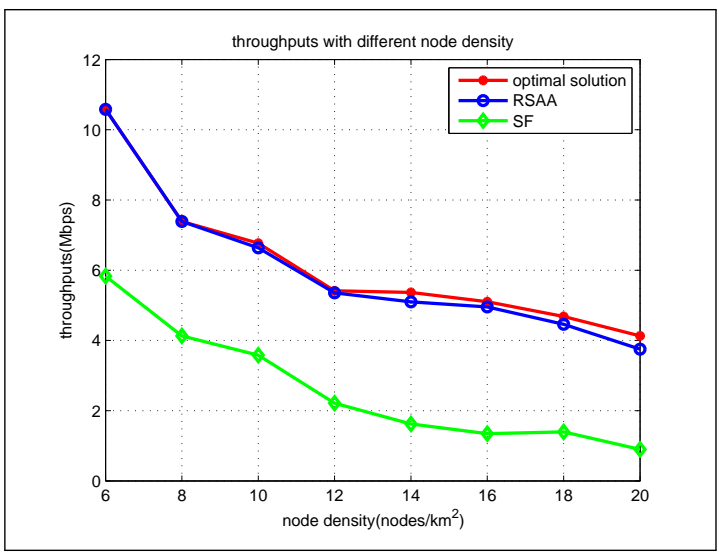

Figure 4: SUs' throughput on different node density.

\subsection{The Effect of Time-Slots and Conflict}

Fig. 5 compares the throughput of SUs got from the three algorithms as the slot periods in the network increase. In this simulation the node density is set as 13 nodes per square kilometer. The PU's threshold $I n_{T}$ and the ratio $R$ are the same with those in Fig. 4. From Fig. 5 we can see that the RSAA's approximation to the optimal solution is not affected by slots and in any slot number condition RSAA can get $98 \%$ of the optimal throughput for SUs. Also we can find that when we fix the length of each slot as 1 second and increase the slot numbers in the network, the SUs' throughput increase dramatically when the number of slot periods is small. If we increase the slot periods from 3 to 5 , SUs' throughput decrease because the increase of data cannot offset the increase of delay. But when the slot period is 6 the throughput of SUs increase as the increase of data outweighs the increase of delay. The fluctuation in Fig. 5 shows the complex relationship between relay and throughput in multi-hop wireless networks.

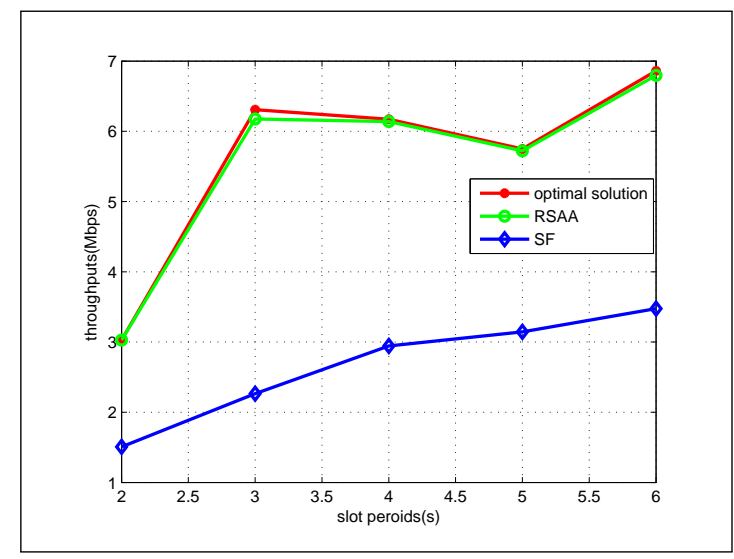

Figure 5: SUs' throughput on different slot periods.

Fig. 6 shows the throughput of SUs vary as the ratio of interference distance to transmitting distance increases under the three algorithms. In this simulation the PU's node density is set as 16 nodes per square kilometer and the PU's threshold is the same as that in Fig. 4. The slot period is 4. From Fig. 6 we can also find that RSAA can get $99 \%$ of the optimal throughput in average compared to SF's $34 \%$. What is more, Fig. 6 shows that as the ratio increases the solutions of RSAA become much closer to the optimal results and when the ratio exceeds 2 , the two solutions nearly overlap with each other which is identical with our above performance analysis. 


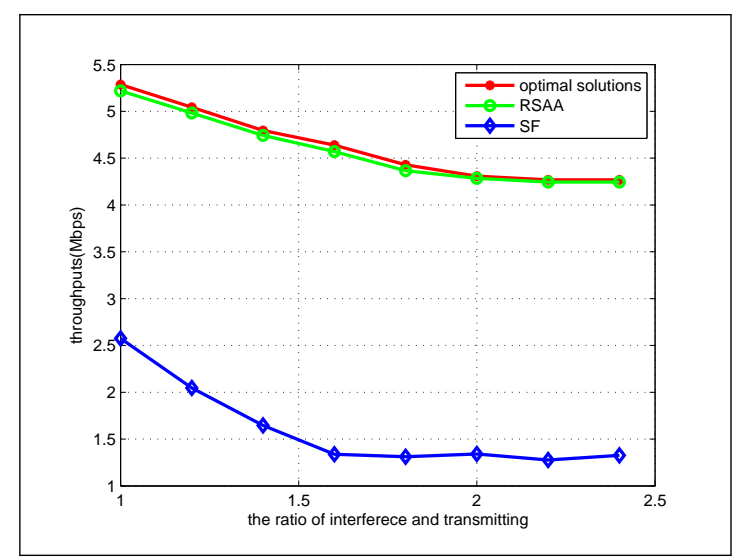

Figure 6: SUs' throughput on different ratio of interference to transmitting.

\section{Conclusions}

In spectrum sharing multi-hop CRNs, by carefully selecting paths and slots SUs can utilize the licensed spectrum band transparently. In this paper, we first formulate a MILP model to describe the joint routing and time-slot assignment issue and then develop RSAA algorithm to solve this NP-Hard problem. Theoretical analyses and simulation results demonstrate that RSAA algorithm can obtain near-optimal throughput with a polynomial complexity and so it can be widely used in CRNs.

\section{Acknowledgment}

This work was supported in part by the National Natural Science Foundation of China (60832007), National Hi-Tech Research and Development Plan of China (2009AA011801), and the National Science and Technology Major Project( 2010ZX03005-003).

\section{Bibliography}

[1] FCC, ET Docket No 03-222 Notice of proposed rule makingand order, December 2003.

[2] J. Mitra III and G. Q. Maguire JR., "Cognitive radio: making software radios more personal," IEEE Personal Commun., pp. 13-18, Aug. 1999.

[3] I. Akyildiz, W. Lee, M. Vuran, and S. Mohanty. "NeXt Generation / Dynamic Spectrum Access/Cognitive Radio Wireless Networks: A Survey." Compo Netw. Jour. (Elsevier), Vol.50, no.13, pp. 21272159, Sept. 2006.

[4] T. Yucek, H. Arslan, "A survey of spectrum sensing algorithms for cognitive radio applications," Communications Surveys \& Tutorials, IEEE, vol.11, no.1, pp.116-130, First Quarter 2009.

[5] H. Wang, H. Qin, L. Zhu, "A Survey on MAC Protocols for Opportunistic Spectrum Access in Cognitive Radio Networks," Computer Science and Software Engineering, 2008 International Conference on , vol.1, no., pp.214-218, 12-14 Dec. 2008

[6] Y. Wang, P. Ren, and G. Wu, "A throughput-aimed MAC protocol with QoS provision for cognitive ad hoc networks," IEICE Trans. Commun., vol. E93-B, no. 6, pp. 1426-1429, Jun. 2010. 
[7] H. Khalife, N. Malouch, S. Fdida, "Multihop cognitive radio networks: to route or not to route," Network, IEEE, vol.23, no.4, pp.20-25, July-August 2009

[8] M. Ceasna, F. Cuomo, E. Ekici, "Routing in cognitive radio networks: Challenges and solutions", Ad Hoc Netw., Vol. 9, no. 3, pp.228-248, May 2011

[9] X. Zhou, L. Lin, J. Wang and X. Zhang, "Cross-layer routing design in cognitive radio networks by colored multigraph model," Wireless Personal Communications, vol. 49, no.1, pp. 123-131, April 2009

[10] Y.T. Hou, Y. Shi, H.D. Sherali, "Optimal Spectrum Sharing for Multi-Hop Software Defined Radio Networks," INFOCOM 2007. 26th IEEE International Conference on Computer Communications. IEEE , vol., no., pp.1-9, 6-12, May 2007

[11] Y.T. Hou, Y. Shi, H.D. Sherali, "Spectrum Sharing for Multi-Hop Networking with Cognitive Radios," Selected Areas in Communications, IEEE Journal on , vol.26, no.1, pp.146-155, Jan. 2008

[12] I. Filippini, E. Ekici, M. Cesana, "Minimum maintenance cost routing in Cognitive Radio Networks," Mobile Adhoc and Sensor Systems, 2009. MASS '09. IEEE 6th International Conference on , vol., no., pp.284-293, 12-15 Oct. 2009

[13] K.R. Chowdhury, I.F. Akyildiz, "CRP: A Routing Protocol for Cognitive Radio Ad Hoc Networks," Selected Areas in Communications, IEEE Journal on, vol.29, no.4, pp.794-804, April 2011

[14] L. Ding, T. Melodia, S.N. Batalama, J.D. Matyjas, M.J. Medley, "Cross-Layer Routing and Dynamic Spectrum Allocation in Cognitive Radio Ad Hoc Networks," Vehicular Technology, IEEE Transactions on , vol.59, no.4, pp.1969-1979, May 2010

[15] M. Xie, W. Zhang, K.K. Wong, "A geometric approach to improve spectrum efficiency for cognitive relay networks," Wireless Communications, IEEE Transactions on, vol.9, no.1, pp.268-281, January 2010

[16] Z. Yuan, J. B. Song, Z. Han, "Interference Minimization Routing and Scheduling in Cognitive Radio Wireless Mesh Networks," Wireless Communications and Networking Conference (WCNC), 2010 IEEE , vol., no., pp.1-6, 18-21 April 2010

[17] P. Gupta, P.R. Kumar, "The capacity of wireless networks," InformationTheory, IEEE Transactions on, vol.46, no.2, pp.388-404, Mar 2000.

[18] K. Jain, J. Padhye, V.N. Padmanabhan, L. Qiu, "Impact of Interference on Multi-Hop Wireless Network Performance", Wireless Networks, Vol 11, no 4, pp. 471-487, July 2005

[19] H.D. Sherali, W.P. Adams, P.J. Driscoll, "Exploiting Special Structures in Constructing a Hierarchy of Relaxations for 0-1 Mixed Integer Problems", Operations Research, Vol.46, no.3, pp.396-405, May 1998

[20] S. Gao. Graph Theory and Network Flow Theory, 1rd ed., BeiJing: Higher Education Press, 2009, pp.307-314

[21] P. Erdfs and A. Renyi, "On the evolution of random graphs", PubI. Math. Inst. Hung. Acad. Sci. 5, 1960, pp.17-61. 